\title{
O estresse dos profissionais de enfermagem em Unidades de Terapia Intensiva: uma revisão bibliográfica
}

\author{
The stress of nursing professionals in Intensive Care Units: a literature review \\ El estrés de los profesionales de enfermería en las Unidades de Cuidados Intensivos: una \\ revisión de la literatura
}

Melina Rodrigues de Souza ${ }^{1 *}$, Pâmella Aparecida Barros ${ }^{1}$, Jussara Camila Ribeiro ${ }^{1}$, Márcio Antonio Resende $^{1,2}$, Hélia Cristina de Souza ${ }^{3}$, Renata Angélica Calsavara ${ }^{3}$, Andréia Andrade dos Santos ${ }^{1}$.

\section{RESUMO}

Objetivo: Consiste em descrever as implicações do estresse na rotina de trabalho dos profissionais de enfermagem nestas unidades. Revisão bibliográfica: Com as inúmeras demandas enfrentadas por enfermeiros em Unidades de Terapia Intensiva, muitos enfermeiros passam a lidar com o estresse, o que interfere no bem-estar, na saúde e no desempenho desses, destacando-se a ocorrência da Síndrome de Burnout, que, por sua vez, se manifesta por meio do esgotamento mental e físico. Diante disso, é importante que esses profissionais adotem estratégias que fortaleçam para o enfrentamento do estresse, consigam preservar sua saúde e desenvolver bem o seu trabalho. Considerações finais: É necessário e imprescindível a adoção de medidas alternativas em prol da saúde dos trabalhadores e da qualidade do trabalho. A aplicação das estratégias de enfrentamento pode ser eficaz no manejo do estresse por meio de intervenções preventivas e protetivas com os profissionais. $O$ alcance da excelência na assistência ao paciente requer condutas e ações que viabilizem um processo dinâmico e sistematizado.

Palavras-chave: Estresse ocupacional, Cuidados de enfermagem, Centro de terapia intensiva.

\begin{abstract}
Objective: It consists of describing the implications of stress in the work routine of nursing professionals in these units. Bibliographic review: With the countless demands faced by nurses in Intensive Care Units, many nurses start to deal with stress, which interferes with their well-being, health and performance, highlighting the occurrence of Burnout Syndrome, which, in turn, manifests itself through mental and physical exhaustion. Given this, it is important that these professionals adopt strategies that strengthen to cope with stress, manage to preserve their health and develop their work well. Final considerations: It is necessary and essential to adopt alternative measures for the health of workers and the quality of work. The application of coping strategies can be effective in managing stress through preventive and protective interventions with professionals. Achieving excellence in patient care requires conduct and actions that enable a dynamic and systematized process.
\end{abstract}

Key words: Occupational stress, Nursing care, Intensive care center.

\section{RESUMEN}

Objetivo: Consiste en describir las implicaciones del estrés en la rutina laboral de los profesionales de enfermería en estas unidades. Revisión bibliográfica: Con las innumerables demandas que enfrentan los

${ }^{1}$ Centro Universitário Presidente Tancredo de Almeida Neves (UNIPTAN). São João Del Rei - MG.

*E-mail: melinarsouza@hotmail.com

2 Universidade Federal de São João Del Rei (UFSJ). São João Del Rei - MG.

SUBMETIDO EM: 11/2019

ACEITO EM: 12/2019

PUBLICADO EM: 3/2021 
enfermeros en las Unidades de Cuidados Intensivos, muchos enfermeros comienzan a lidiar con el estrés, el cual interfiere con su bienestar, salud y desempeño, destacando la ocurrencia del Síndrome de Burnout, que a su vez se manifiesta a través de agotamiento mental y físico. Ante esto, es importante que estos profesionales adopten estrategias que fortalezcan para hacer frente al estrés, logren preservar su salud y desarrollen bien su trabajo. Consideraciones finales: Es necesario e imprescindible adoptar medidas alternativas para la salud de los trabajadores y la calidad del trabajo. La aplicación de estrategias de afrontamiento puede ser eficaz en el manejo del estrés mediante intervenciones preventivas y protectoras con profesionales. Alcanzar la excelencia en la atención al paciente requiere de conductas y acciones que permitan un proceso dinámico y sistematizado.

Palabras clave: Estrés laboral, Cuidados de enfermería, Centro de cuidados intensivos.

\section{INTRODUÇÃO}

A prática do enfermeiro se reúne a uma complexa trama de situações relacionadas à assistência direta ao paciente e aos fatores da organização do trabalho que contribuem para a ocorrência de estresse, gerando perturbações e comprometendo a qualidade da assistência a ser prestada (OLIVEIRA EM, et al., 2011). A exposição prolongada aos agentes estressores tem como consequência baixo desempenho profissional, moral e baixa autoestima, alta rotatividade, absenteísmo e violência, prejudicando todo o trabalho realizado. A enfermagem tem sido considerada uma das profissões que mais tem sofrido impacto do estresse em seu ambiente de trabalho (GUIDO LA, et al., 2011).

Nas Unidades de Terapia Intensiva (UTI), setor fechado, que tem por finalidade a reabilitação mais rápida, em um local à parte do hospital com recursos materiais e profissionais adequados é de suma importância a presença da enfermagem para gerenciar e assistir o paciente de forma humanizada, considerado uma unidade altamente estressora por causa dos momentos emergenciais e complexos (PEDRÃO J e PRETO $A V, 2009)$. Por se tratar de um termo comumente conhecido entre os profissionais de enfermagem que trabalham na UTI, o estresse tem ocupado lugar de destaque em meio à globalização e das novas tecnologias, passando a ser considerada o "mal do século" pelos cientistas (DECEZARO A, et al., 2014).

O estresse é definido como uma resposta física do nosso organismo a um estímulo físico, emocional ou comportamental ao qual o indivíduo é exposto e que gera desgaste (PEDRÃO J e PRETO AV, 2009). Além de estar diariamente presente na vida das pessoas, o estresse tem aumentado seus fatores desencadeantes frente aos trabalhadores, desde as tarefas mais simples realizadas, àquelas que podem exigir uma demanda maior de esforço físico ou mental, atingindo um número cada vez mais significativo de pessoas que se dizem estressadas ou que relatam conhecer pessoas nessa situação (SILVA LC e SALLES CTA, 2016).

Considerando que o exercício da enfermagem gera um clima de grande tensão emocional, desgaste físico e psíquico, no qual pode contribuir como fator desencadeante do stress. Isso exigiria, também, do profissional enfermeiro uma adaptação em relação a esses agentes estressores para manter o seu equilíbrio homeostático (ALVES ACJC, 2011).

Para Pedrão J e Preto AV (2009), o enfermeiro tem exigências dentro das unidades de saúde que levam ao estresse, pois o mesmo resolve diversos problemas institucionais deixando de lado suas necessidades individuais. Um dos grandes motivos de absenteísmo na equipe de enfermagem está o afastamento ligado a doenças ocupacionais, a licença médica. Tal situação corriqueira nas instituições de saúde implica na necessidade de uma maior atenção dos gestores de saúde e pesquisadores, principalmente quando se trata de profissionais da área de terapia intensiva (GRILLO PDP, 2019).

Dessa forma, tanto a Enfermagem gerencial quanto assistencial necessita dedicar-se na implantação de medidas que visem à segurança através de informação e conhecimento. Uma abordagem sobre a comunicação multiprofissional e intersetorial efetiva na instituição se mostram indispensáveis (LINCH CGF e GUIDO LA, 2011). 
Este estudo traz como objetivo descrever e revisar as implicações do estresse na rotina de trabalho dos profissionais de saúde que atuam nas UTI's.

\section{REVISÃO BIBLIOGRÁFICA}

\section{O estresse na rotina da enfermagem}

Conhecido por fazer parte da realidade dos profissionais de enfermagem, o estresse é considerado uma doença moderna que possui agentes estressores diversos, sintomas exaustivos e perceptíveis em indivíduos que lidam em ambientes de trabalho desgastantes, trazendo grandes consequências para a saúde física, psíquica e mental (LINCH CGF e GUIDO LA, 2011).

Agentes estressores são denominações dadas a eventos geradores de exaustão emocional e física, podendo se diferenciar nos sintomas apresentados de uma pessoa para outra. Algo que amedronte, excite, atrapalhe, desgaste ou confunda o indivíduo pode ser considerado um agente estressor (MIQUELIM JDL, 2004).

O estresse em enfermeiros é um tema muito discutido e investigado contemporaneamente. Estudos revelam que estes profissionais enfrentam cargas elevadas de pressão psicológica, o que a longo prazo pode desencadear diversos problemas de saúde (BATISTA KM e BIANCHI ERF, 2006).

Em se tratando de trabalho, se consegue imaginar vários fatores que podem de alguma forma, torná-lo estressante. Ainda mais em algumas profissões em que o trabalho é sobre pressão, cobranças, metas, barulho constante, incertezas e dificuldades, propicia ao trabalhador um maior desgaste físico e mental (RODRIGUES AB e CHAVES EC, 2013).

Percebe-se que no decorrer do tempo, mais precisamente nas últimas décadas têm-se notado um crescimento do estresse nos ambientes de trabalho, tornando algo avassalador na vida do indivíduo, trazendo insatisfação, desinteresse, apatia e irritação. Para que se evite estresse, o ideal é que este trabalho possa oferecer harmonia, precisa que seja algo prazeroso, com requisitos mínimos para a atuação e para a qualidade de vida dos indivíduos (BATISTA KM e BIANCHI ERF, 2006).

O perfil emotivo dos profissionais atuantes dentro de UTIs se altera durante a jornada diária de trabalho, desgastando e estressando pela repetição de tarefas que exigem alto conhecimento teórico e prático, além da demanda de agilidade (PEDRÃO JL e PRETO AV, 2009).

O estresse ocupacional ou relacionado ao serviço do enfermeiro gera prejuízo ao bem-estar psicossocial do indivíduo, relacionado, por vezes, a alterações no estado de saúde. E, ainda, pode colocar em risco a saúde dos membros da organização e pode gerar diminuição no rendimento do trabalho, autoestima baixa e depressão (RODRIGUES AB e CHAVES EC, 2008).

Para os profissionais de enfermagem, o trabalho, se diz dignificante, engrandece o ser por suas ações, em contradição os indivíduos enfrentam uma realidade dura, um ambiente estressante, de barulhos constantes, mortes, sofrimento, desespero, irritabilidade, ansiedade, dores ininterruptas, medo e insegurança (ALVES ACJC, 2011). A complexidade da assistência de enfermagem em situações que envolvem o cuidado, assim como a assistência e o gerenciamento da equipe são considerados desgastantes, tanto pela especificidade de suas tarefas quanto pelo fato de lidar com as tristezas e sofrimentos constantemente (BATISTA KM e BIANCHI ERF, 2006). No processo de organização do trabalho e nos procedimentos realizados em todos os setores de um hospital, há evidências de exposição contínua dos enfermeiros a situações e fatores do estresse, nas dimensões técnicas, institucionais e interpessoais que poderão influenciar no processo de exaustão nesses profissionais (ALVES ACJC, 2011).

A construção da escala de plantão da equipe de enfermagem para atuar na UTI é considerado fator estressante. É complicado montar o plantão em épocas de feriado, folgas, licença maternidade, férias pois a equipe vai trabalhar em um número menor de profissionais, que também é considerado estressor pela sobrecarga de trabalho ou será necessário o remanejamento de outro plantão. É importante salientar que 
vários enfermeiros atuam em mais de uma unidade então tem que tomar cuidado para não bater os plantões (PEREIRA JG e MELLO F, 2016).

Considera-se que o sujeito poderá sofrer consequências psíquicas por um tempo longo, se dentro do ambiente de trabalho o estresse for avançando e não tratado. Logo, se pode pensar em uma demanda cada vez maior, pois tem se tornado cada vez mais comum surgirem matérias nos jornais e revistas de profissionais fatigados, adoecidos jovens na área da saúde, afastados por doenças do trabalho, altos índices de absenteísmo causados pela Síndrome de Burnout (MARTINATO CNB, et al., 2011). Estudos mostram que o desequilíbrio na saúde do profissional pode levá-lo a se ausentar do plantão aumentando gastos com tratamento de saúde e necessidades da instituição com reposição de funcionários, transferências, novas contratações, novo treinamento, entre outras despesas. $O$ atendimento, lucratividade e satisfação e lucratividade tendem a diminuir (STRAUB RO, 2019).

Daí surge a importância de compreender a prática diária da equipe de profissionais dentro da UTI, identificando as dificuldades enfrentadas dentro de cada plantão para o gestor da equipe agir precocemente nos fatores estressantes melhorando a qualidade de saúde de sua equipe e garantir maior segurança aos pacientes (PEDRÃO JL e PRETO AV, 2009). Diante do exposto, cabe ressaltar que a resposta ao estresse é individual, pois como o indivíduo absorve a informação e a sua avaliação sobre estímulos classificados por ele como relevantes, sejam eles agradáveis ou amedrontadores, porque uma vez que se escolhe um ambiente de trabalho estressante, sobrecarregado, de ambiente insalubre, em regime de plantões duplos e triplos, baixa remuneração, o indivíduo está sujeito a uma série de problemas futuros bem como adoecimento e sofrimento psíquico, podendo levar a uma Síndrome de Burnout (SANTOS JW, 2009).

\section{A Síndrome de Burnout nas Unidades de Terapia Intensiva}

A UTI é o ambiente adequado para atender pacientes críticos, de gravidade, por isso considerado ambiente tenso, o qual afeta não somente o paciente, como também toda a equipe que atua nesse setor das Instituições de maior complexidade (SANTOS FE, et al., 2010). A Síndrome de Burnout é uma condição associada ao esgotamento mental, físico e emocional, que ocorre como resultado de estresse excessivo a longo prazo. $O$ esgotamento pode fazer com que uma pessoa se sinta sobrecarregada e incapaz de lidar com as demandas constantes da vida cotidiana (OLIVEIRA MC, 2018).

Para Souza AMJ, et al. (2018), a Síndrome de Burnout se transformou num problema de saúde pública, pois integra o rol de doenças ocupacionais, tendo como característica a percepção de esgotamento físico, mental e emocionalmente, por conta do trabalho. Nesse sentido, é compreendido como problema de saúde ocupacional que faz com que a pessoa se sinta despersonalizada e pouco realizada profissionalmente. $O$ Ministério da Saúde descreve a Síndrome de Burnout como "síndrome do esgotamento profissional" em consequência da resposta estressora e prolongada de estresse laboral e de insatisfação plena no ambiente de trabalho (MATUBARO KCA, et al., 2013).

A síndrome é uma experiência individual que prejudica a relação do indivíduo com seu trabalho, diminui a atuação, gerando baixo rendimento para a instituição, trabalhador e pode estender-se para o usuário do serviço. Assim, tornou-se uma importante preocupação e é reconhecido como um dos riscos mais sérios ao bem-estar psicossocial do indivíduo, uma vez que interfere significativamente como fator determinante de outras patologias, como aos transtornos depressivos e muito frequentemente, estes profissionais evoluem para o desenvolvimento da Síndrome de Burnout (SILVA FD, et al., 2016).

Em se tratando dos profissionais da Unidade de Terapia Intensiva, como todo profissional que lida com a urgência, é preciso ficar atento aos limites do trabalho e em qualquer sinal de estresse. Suas atribuições são executadas sobre forte pressão, associada a longas jornadas e em turnos, com rodízio e multiplicidade de tarefas, marcado pela repetição de atividades, esforço corporal, posição que favorece o aparecimento de dores, agitação e falta de liderança e trabalho em equipe causa o desencadeamento de doenças (NEVES $\mathrm{RR}, 2008)$.

Estudos revelam que os trabalhadores da área da saúde, que atuam em instituições hospitalares estão frequentemente expostos aos estressores ocupacionais, chegando a representar o grupo de trabalhadores 
mais afetado, mostrando taxas de Burnout que variam entre 30 e 47\% (RUVIARO MFS e BARDAGI MP, 2010). Dentre as principais causas desencadeadoras de estresse podemos citar o excesso de atividades, pouco tempo para se realizar várias tarefas (pessoais e profissionais), pressões e cobranças, acúmulo de raiva, desvalorização, alta competitividade, podendo assim atingir qualquer pessoa (SILVA LC e SALLES CTA, 2016).

O nível de estresse se difere de um indivíduo para com o outro, ou seja, dentro de uma Unidade de Terapia Intensiva, o trabalho pode ser mais estressante para um profissional da enfermagem, do que para outro, dependendo principalmente do ritmo de vida de cada um e da sua jornada de trabalho. Diante disso, diversas são as medidas e estratégias de intervenção, para auxiliar a lidar com os agentes estressores sem prejuízos para a saúde do enfermeiro (PEREIRA JG e MELLO F, 2016).

Assim, a adoção de estratégias eficazes no combate ao estresse depende das características individuais do profissional e de situações vivenciadas no ambiente ocupacional, pois desta maneira se pode permitir que a enfermagem na gestão dos seus colaboradores pode promover recursos para enfrentar a situação estressante ao aderir a várias delas (SOUZA FD, et al., 2018)

\section{Estratégias de intervenção para prevenir e tratar o estresse no trabalho}

Quando se identifica um agravo sugestivo de síndrome, seja ela de qualquer origem, faz-se necessário acompanhamento com ajuda de um profissional, sendo adotadas estratégicas, pois identificados sinais sugestivos, como baixa produção e menor qualidade no atendimento prestado aos seus pacientes, é preciso acompanhar o indivíduo a fim de que ele se recupere e I se torne apto a realizar todas as suas funções e sem ter seu estado emocional afetado (PEREIRA DG, 2011).

Uma importante estratégia para minimizar o estresse no trabalho é contar com o apoio e acompanhamento do enfermeiro Responsável Técnico da Unidade de terapia Intensiva, competente principalmente na avaliação e encaminhamento do profissional adoecido para tratamento especializado, afastamento das suas atividades ou ainda remanejamento de setor (PEREIRA JG e MELLO F, 2016).

Uma das modalidades aplicadas no processo de enfermagem no combate ao estresse são as estratégias de enfrentamento, que podem ser classificadas como cognitivas, comportamentais ou emocionais que visam lidar com os agentes estressores minimizando os seus efeitos nocivos ao organismo do profissional no exercício de suas atividades no ambiente de trabalho (STRAUB RO, 2014). Identificado como uma estratégia de enfrentamento eficaz, o afastamento temporário do ambiente de trabalho faz com que trabalhadores de enfermagem se distanciem da atividade no setor por alguns momentos, como forma de distração e reorganização psicológica frente ao problema causador do estresse (PEREIRA CA, et al., 2009).

O conhecimento sobre as estratégias de enfrentamento das suas emoções e sentimentos que os profissionais de enfermagem utilizam para se adaptarem ao estresse pode direcionar as ações dos enfermeiros e gestores na busca da resolução do problema, levando a um ambiente de trabalho com menos problemas e mais saudável. Enfermeiros e gestores devem planejar os cuidados em saúde, realizar encaminhamento destes profissionais adoecidos para dinâmicas de trabalho, para setores da medicina do trabalho e tratar interdisciplinarmente as dificuldades identificadas. $O$ acompanhamento, a escuta, os programas educacionais e um espaço para discussão das dificuldades relacionadas com o estresse colaboram para a prevenção do adoecimento e a promoção da qualidade do trabalho (RIBEIRO RM, et al., 2015).

A enfermagem sente-se protegida e amparada à medida que cuidados lhe são oferecidos no ambiente de trabalho, em condições sociais e estruturais, dignas de conforto, bem-estar, realização e valorização pessoal e profissional. $O$ trabalho e as atividades propostas devem priorizar as condições humanas potencialmente, adaptando a organização e possibilitando a expressão de suas emoções e pontos de vista (SILVA LC e SALLES CTA, 2016).

Com intuito de minimizar e combater o surgimento da síndrome de Burnout, uma alternativa viável para com os profissionais seria a prática de inserir programas de promoção à saúde com acompanhamento dos 
funcionários, dimensionamento de pessoal nas áreas adequadas, avaliações constantes sobre como está a satisfação com o trabalho e a comunicação entre os componentes da equipe, o que causará satisfação e gerará um maior desempenho na assistência prestada por estes profissionais (XAVIER LMR, et al., 2019). Outra estratégia interessante a ser trabalhada no controle do estresse seria para o gerente ou responsável do setor de Recursos Humanos monitorar cada indivíduo e adequar particularmente este com o setor que mais compete a ele, identificando e trabalhando os pontos de especialidade e organizando suas atribuições ao setor que combina com seu perfil, ou seja, adequar cada profissional com o cargo ideal, bem como propor um planejamento de carreira (LARRÉ MC, et al., 2018).

O apoio religioso foi identificado por diversos autores como eficaz em um estudo realizado com enfermeiros da UTI, pois o apego a uma crença religiosa auxilia no equilíbrio emocional, a lidar com pontos de equilíbrio emocional durante uma situação estressante. $O$ apoiamento na cultura religiosa é positivo tanto para 0 paciente quanto para o profissional interferindo na empatia e dimensionando questões existenciais (MIORIN JD, et al., 2016). Demais terapias alternativas também podem ser aplicadas no combate ao estresse no ambiente de trabalho como forma humanizada de tratamento, evitando o consumo de drogas psicoativas, que demandam tempo e efeitos colaterais, como floral de Bach e Reiki, a leitura de livros de autoajuda, práticas de meditação e relaxamento, massagens e apoio familiar (SOUZA RC, et al., 2018).

A busca por tratamentos não convencionais como a hipnose, atividades físicas e a acupuntura também tem sido considerado essencial para atuar no controle do estresse, podendo ser escolhido pelo profissional da enfermagem de acordo com sua necessidade e pode acontecer fora do ambiente de trabalho, de forma segura e diversificada, sem preconceitos (SILVA LC e SALLES CTA, 2016).

A hipnose em situações relacionadas ao trabalho auxilia na redução do estresse, na melhoria da relação entre os colaboradores, dentre outros e podem ser usadas de diversas maneiras, como nas consultas psicanalistas, em cursos, seminários e palestras (PUENTES F e TESSER O, 2014). A prática regular de atividade física demonstra-se como um componente indispensável na prevenção e manutenção da saúde dos trabalhadores, assim como para todo indivíduo, por proporcionar melhor qualidade de vida e auxiliar no combate a doenças decorrentes do sedentarismo e da vida atribulada, os quais podem ser prejudiciais à saúde física e mental (GORSKI GM, 2015).

Estudos comprovam que a variável atividade física apresentou impacto no estresse ocupacional, de forma que o nível de estresse foi maior nos indivíduos que não praticavam atividade física, caracterizando-a como uma forma de minimizar possíveis estressores no ambiente de trabalho, enquanto estudo semelhante reforça a relação entre atividade física nos momentos de lazer e estresse ocupacional de 861 trabalhadores e identificou que realizar atividade física nos momentos de lazer ajuda a minimizar altos níveis de estresse no trabalho, e a longo prazo, melhora o bem-estar mental (CANOVA RK e PORTO GB, 2010).

De acordo com Ribeiro RM, et al. (2015), pontos como liderança, gerenciamento, trabalho em equipe e comunicação efetiva são fundamentais para o profissional de enfermagem dentro da UTI por ter que agir rapidamente, podendo ser assuntos tratados em formações continuadas. Assim sendo, a utilização dessas estratégias pode fortalecer o indivíduo e a equipe de Enfermagem no enfrentamento de estressores, facilitar a convivência entre os membros, desenvolver habilidades e promover a motivação e a satisfação no trabalho. Se tratado, o profissional poderá seguir suas atividades no ambiente hospitalar com satisfação e serenidade na Instituição.

\section{CONSIDERAÇÕES FINAIS}

O conhecimento sobre os fatores estressantes dos profissionais da enfermagem dentro da UTI é fundamental para o enfermeiro gestor apontar alternativas que auxiliam na minimização dos fatores desencadeadores do estresse e diminuir o impacto na rotina dos profissionais de enfermagem, demonstrando a importância de se cuidar da saúde física e mental e melhorar a qualidade da assistência e do atendimento aos pacientes. A aplicação das estratégias de enfrentamento pode ser eficaz no manejo do estresse por meio de intervenções preventivas e protetivas com os profissionais. $O$ alcance da excelência na assistência ao 
paciente requer condutas e ações que viabilizem um processo dinâmico e sistematizado capaz, antes de estudo, garantir a saúde e o bem-estar da equipe de enfermagem.

\section{REFERÊNCIAS}

1. ALVES ACGC. Estresse e o trabalho do enfermeiro: uma revisão bibliográfica. 2011. Trabalho de Conclusão de Curso (Especialização em Gestão de Sistemas e Serviços de Saúde) - Centro de Pesquisa Aggeu Magalhães, Fundação Oswaldo Cruz, Recife; 2011;26p.

2. BATISTA KM, BIANCHI ERF. Estresse do enfermeiro em unidade de emergência. Rev. Latino-Am. Enfermagem, 2006; 14(4).

3. CANOVA KR, PORTO JB. O impacto dos valores organizacionais no estresse ocupacional: um estudo com professores de ensino médio. Revista de Administração Mackenzie, 2010; 11(5).

4. CUNHA T. Estresse do profissional de saúde no ambiente de trabalho: causas e consequências. Caderno Saúde e Desenvolvimento, 2019; 3(2): 78-93.

5. DECEZARO A, et al. O estresse dos enfermeiros que atuam na unidade de terapia intensiva: uma revisão de literatura. Revista Unigá, 2014; 19(2): 29-32.

6. GORSKI GM. Relação entre atividade física de lazer e Burnout em trabalhadores da indústria. 2015. Dissertação (Mestrado em Engenharia da Produção) - Universidade do Paraná, Ponta Grossa; 2015.

7. GRILLO PDP. Absenteísmo em profissionais da área de enfermagem: causas mais frequentes - uma revisão da bibliografia atual. Rev Sau Era, 2019; 2(1):15-20.

8. GUIDO LA, et al. Estresse, coping e estado de saúde entre enfermeiros hospitalares. Revista da Escola, 2011; 45(6).

9. LARRÉ MC, et al. A relação da Síndrome de Burnout com os profissionais de enfermagem: revisão integrativa. Nursing, 2018; 21(237): 2018-2023.

10. LINCO CGF, GUIDO LA. Estresse de enfermeiros em unidade de hemodinâmica no Rio Grande do Sul, Brasil. Revista Gaúcha de Enfermagem, 2011; 32(11).

11. MARTINATO MCNB, et al. Absenteísmo na enfermagem: uma revisão integrativa. Rev Gaúcha Enferm., 2010; 31(1).

12. MATUBARO KCA, et.al. Síndrome de Burnout em profissionais da saúde: uma revisão bibliográfica. Psicologia, 2015; $1(1)$.

13. MIORIN JD, et al. Estratégias de defesa utilizadas por trabalhadores de enfermagem atuantes em pronto socorro. Revista Enferm Foco, 2016; 7(2).

14. MIQUELIM JDL. Estresse nos profissionais de enfermagem que atuam em uma unidade de pacientes portadores de hiv-aids. DST - J bras Doenças Sex Transm, 2004; 36(91).

15. NEVES RR. As profissões mais estressantes. Globoplay (Editora Globo), 2008. Disponível em: $\mathrm{https://globoplay.globo.com/v/884658/.} \mathrm{Acesso} \mathrm{em:} 20 \mathrm{dez} .2020$.

16. OLIVEIRA MC. Síndrome de Burnout e os fatores de risco em profissionais de enfermagem. 2018. Trabalho de Conclusão de Curso (Bacharelado em Enfermagem) - Universidade Católica do Salvador - UCSAL. Salvador, 2018.

17. OLIVEIRA EM. Qualidade de vida no trabalho. Rio de Janeiro: Brasport; 2011.

18. PEDRÃO JL, PRETO AV. O estresse entre enfermeiros que atuam em Unidade de Terapia Intensiva. Revista da escola de enfermagem da USP, 2009; 43(4): 842.

19. PEREIRA DG. Síndrome de Burnout em trabalhadores do Programa de Saúde da Família: uma revisão de literatura. 2011. Trabalho de Conclusão de Curso (Especialização em Atenção Básica em Saúde da Família) - Universidade Federal de Minas Gerais. Faculdade de Medicina. Núcleo de Educação em Saúde Coletiva, 2011. 37p.

20. PEREIRA JG, MELLO F. Causas e efeitos do estresse no trabalho. Interação, 2016; 16(16):146-164.

21. PEREIRA CA, et al. O estresse ocupacional da equipe de enfermagem em setor fechado. J Res Fund Care, 2009; $1(2)$.

22. PUENTES F, TESSER O. Hipnose no trabalho, Freud explica. São Paulo: Saraiva; 2011.

23. RIBEIRO RM, et al. Estratégias de enfrentamento dos enfermeiros em serviço hospitalar de emergência. Acta Paul Enferm, 2015; 28(3): 216-23.

24. RODRIGUES AB, CHAVES EC. Fatores estressantes e estratégias de coping dos enfermeiros atuantes em oncologia. Revista Latino-Americana de Enfermagem, 2008; 16(1).

25. RODRIGUES DP, et al. Estresse na Unidade de Terapia Intensiva. Rev enferm UFPE, 2013; 7(esp):1595-604

26. RUVIARO MFS, BARDAGI MP. Síndrome de Burnout e satisfação no trabalho em profissionais da área de enfermagem do interior do RS. Revista Barbarói, 2010; 1(33).

27. SANTOS FD, et al. O estresse do enfermeiro nas unidades de terapia intensiva adulto: uma revisão de literatura. SMA, 2016; 6(1).

28. SANTOS FE, et al. Síndrome de Burnout em enfermeiros atuantes em uma Unidade de Terapia Intensiva. Einstein, 2010; 7(1): 58-63.

29. SANTOS JW. A Síndrome de Burnout: uma análise social e psicodinâmica. Revista Científica Eletrônica de psicologia. São Paulo: FAEF; 2009.

30. SILVA ABN, et al. Síndrome de Bournot em profissionais de enfermagem na Unidade de Terapia Intensiva. Revista Ciência Saúde Nova Esperança, 2016; 14(1): 79-86.

31. SILVA LC, SALLES CTA. O estresse ocupacional e as formas alternativas de tratamento. ReCaPe - Revista de Carreiras e Pessoas, 2016; 6(2).

32. SOUZA RC, et al. Estratégias de enfrentamento do estresse. Revista Brasileira de Medicina do Trabalho, 2018; 30(3): 147-153.

33. SOUZA AMJ, et al. Síndrome de Burnout: Fatores de risco em enfermeiros de unidades de terapia intensiva. C\&DRevista Eletrônica da FAINOR, 2018; 11(2): 304-315.

34. STRAUB RO. Psicologia da saúde: uma abordagem biopsicossocial. Porto Alegre: Artmed; 2014.

35. XAVIER LMR, et al. Síndrome de Burnout: Como vencer? Revista Eletrônica Anima Terra, 2019; 9(5): 29-44. 